10.1071/NB07094_ER

Erratum. In the Communicable Diseases Reports for July and August (N SW Public Health Bull 2007; 18(7-8): 145) and September and October (N S W Public Health Bull 2007; 18(9-10): 205), the data in the graphs 'Gastroenteritis outbreaks in institutions' are incorrect as the $x$-axis (year) was incorrectly labelled. The following corrections for these graphs should be noted.
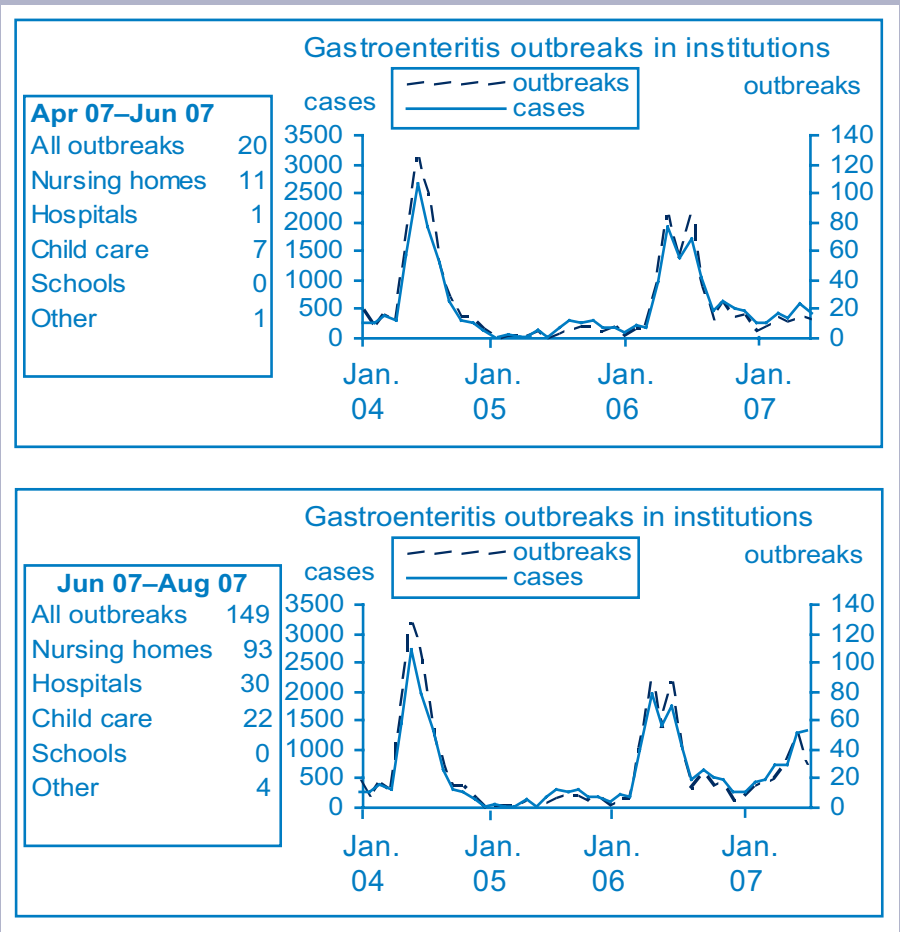


\section{Communicable Diseases Report, New South Wales, May and June 2007}

\section{Communicable Diseases Branch, NSW Department of Health}

For updated information, including data and facts on specific diseases, visit www.health.nsw.gov.au and click on Infectious Diseases.

Figure 3 and Tables 1 and 2 show reports of communicable diseases received through to the end of May and June 2007 in NSW.

\section{Meningococcal disease}

Eight cases of meningococcal disease were notified in NSW in each of May and June. The number of case reports of meningococcal disease has fallen steadily in NSW from 253 in 2000 to 104 in 2006 . As the peak season for this disease is approaching, NSW Health recently faxed general practitioners the following key messages:

- Early diagnosis and treatment by GPs can be lifesaving: benzylpenicillin is the treatment of choice for suspected cases, preferably intravenously

- Patients should be reassessed when the diagnosis is uncertain

- Meningococcal C conjugate vaccine is still free for people up to 25 years of age who were not vaccinated during the school vaccination program.

A biweekly review of meningococcal disease, as well as treatment and prevention guidelines, appears at the above website.

\section{Influenza}

Influenza activity increased markedly in the second half of June to moderate levels, as measured by an upturn in the rate of patients attending emergency departments and the number of respiratory specimens from patients that have tested positive for influenza A.

Influenza symptoms include: fever, headache, myalgia, lethargy, coryza, sore throat and cough. Children may also have nausea, vomiting and diarrhoea.

Treatment is largely supportive although neuraminidase inhibitors can decrease the severity and duration of the illness if treatment is commenced within the first 48 hours of the onset of illness.

To help reduce its spread, people with influenza are encouraged to:

- Use tissues and cover the mouth and nose when coughing or sneezing

- Wash hands frequently with soap and running water for 10 seconds, especially after coughing or blowing the nose

- Stay away from school, work and crowded places while unwell.

Influenza vaccine is recommended for:

- All people aged 65 years and older

- Aboriginal people 50 years and older, and younger Aboriginal adults with medical risk factors

- Children (over six months of age) and adults with chronic illnesses

- Residents of nursing homes and other long-term care facilities

- Contacts of high-risk patients.

Influenza vaccination is not recommended by the National Health and Medical Research Council (NHMRC) as a universal population-based vaccination program for children; however, it can be given to people over six months of age who wish to reduce their likelihood of becoming ill with influenza. Full protection is usually achieved within 10 to 14 days of vaccination.

For details, see the weekly review of influenza activity at the above website.

\section{Legionnaires' disease}

Seven cases of legionnaires' disease due to infection with Legionella pneumophila were reported in May and four in June, including two people who died. None of the cases were linked. The number of cases is in line with seasonal expectations.

\section{Enteric diseases \\ May}

Eight outbreaks of food-borne disease were investigated by NSW Public Health Units in May 2007. These outbreaks affected between two and 17 people each, and were linked to four restaurants, three cafés and one aged-care facility. In one outbreak, reported by Sydney West Area Health Service (Penrith office), Salmonella infection was 
identified as the cause of the illness affecting nine people whose common exposure was eating deep-fried ice cream at a restaurant. Further investigation of the source of ingredients used in the deep-fried ice cream is underway.

There were 27 outbreaks of gastrointestinal disease in institutions reported in NSW in May. These outbreaks included 17 aged-care facilities, five child-care centres, two hospitals and one school. Viruses were confirmed as the cause in seven outbreaks (rotavirus in one and norovirus in six), and the remaining 20 had clinical and epidemiological features consistent with viral gastroenteritis. These features can include nausea, vomiting, diarrhoea, fever, abdominal pain, headache and muscle aches and a high secondary attack rate.

Norovirus is the most commonly reported cause of diarrhoeal outbreaks in aged-care facilities in NSW, and rotavirus is a common cause of viral gastroenteritis outbreaks in child-care centres. Control depends on early recognition that an outbreak is occurring; exclusion (or isolation) of infectious cases; careful hand washing; care with food preparation and environmental cleaning.

For more information about norovirus, see:

http://www.health.nsw.gov.au/infect/pdf/viral_gastro.pdf.

For information about rotavirus see:

http://www.ncirs.usyd.edu.au/facts/rotavirus_vaccine_

for_children_sep_2006.pdf

For guidelines on managing outbreaks in institutional settings see: http://www.health.nsw.gov.au/pubs/2004/ gastroctrl_fs.html.

\section{June}

NSW Public Health Units investigated nine suspected food-borne disease outbreaks in June. These outbreaks were reported to affect between two and 10 people and occurred after people had eaten food from a café, restaurant or takeaway outlet. Scombroid poisoning was suspected to be the cause of illness in two people who ate grilled tuna. Their symptoms resolved following treatment with antihistamines. In most of the other outbreaks, there was insufficient evidence to identify specific pathogens or food items causing illness, and viral infection remains a likely cause for many of the illnesses.

Twenty outbreaks of gastroenteritis in institutions affecting more than 350 people were notified in June. The settings for these outbreaks were: aged-care facilities (11), child-care facilities (7), a hospital and a residential institution. Norovirus was confirmed to be the cause of four of the outbreaks, all in aged-care facilities. Although viral gastrointestinal disease in institutions tends to increase during winter, the rates of illness appears to be lower this year compared to 2006 .

\section{Statewide outbreak of cryptosporidiosis in NSW,} November 2005-May 2006

\section{Kerri A. Viney and Jeremy M. McAnulty \\ Communicable Diseases Branch, NSW Department of Health}

Cryptosporidiosis is a diarrhoeal disease caused by the intestinal parasite, Cryptosporidium. ${ }^{1}$ There are several species of cryptosporidium, but two species, Cryptosporidium parvum and Cryptosporidium hominis, are thought to be responsible for the majority of infections in humans. ${ }^{2}$ Humans are thought to be the major reservoir for C. hominis, while C. parvum infects humans and a range of animals including livestock. ${ }^{3}$

The major symptom of cryptosporidiosis is diarrhoea, which may be profuse and watery and is often associated with cramping abdominal pain. The incubation period is one to 12 days and a person with cryptosporidiosis may excrete oocysts for several weeks after symptoms resolve. ${ }^{1}$ The mode of transmission is the faecal-oral route and includes animal-to-person, person-to-person, water-borne and rarely, food-borne transmission. ${ }^{1}$ Outbreaks have been associated with swimming pools, drinking water supplies and, occasionally, consumption of contaminated beverages. ${ }^{1}$

Cryptosporidium has been notifiable to the NSW Health Department since 1996, and several outbreaks linked to swimming in contaminated pools have been identified. ${ }^{4-7}$ Several hundred cases of cryptosporidiosis are reported in NSW each year and the number of cases tends to increase in the warmer months.

In November 2005, analysis of routinely collected notification data by NSW Health identified a sharp increase in the number of people with cryptosporidiosis: 114 cases were reported in November, up from 28 in October. Initial interviews with cases indicated that an unusually high number $(28 \%)$ had reported contact with animals or farms. We received anecdotal reports from a NSW District veterinarian of concurrent cryptosporidiosis in calves with considerable numbers of oocysts detected in the faeces of sick calves. NSW Health subsequently issued a media release warning of the risk of cryptosporidiosis following contact with farm animals, urging people to take care with hygiene after animal contact, and (to avoid contamination of swimming pools) advising people not to swim if they had had diarrhoea in the previous week. To identify the reasons for the increase in notifications, we initiated a descriptive study of cases in NSW notified between 1 November 2005 and 29 May 2006.

\section{Methods}

A case of cryptosporidiosis was defined as a resident of NSW who had a laboratory confirmed case of cryptosporidiosis, and who was notified between 1 November 
2005 and 29 May 2006. Demographic characteristics of notified cases were routinely entered into NSW Health's Notifiable Diseases Database (NDD). We asked NSW Public Health Units to interview all cryptosporidiosis cases notified between 1 November 2005 and 29 May 2006 with a standard questionnaire in order to identify potential risk factors including attendance at preschool, contact with another case, contact with pets and farm animals and farm visits. These data were entered into Net Epi Case Manager, a web-based database developed by the Centre for Epidemiology and Research (NSW Health). ${ }^{8}$ NDD and Net Epi data were then analysed using SAS. ${ }^{9}$ We analysed cases by exposure, date of onset, report date, area of residence, gender and age group. We ranked exposures in the following order: contact with farm animals (including farm visits), swimming, contact with another case and other exposure (such as preschool attendance, contact with pets and drinking untreated water).

\section{Results}

In December 2006, 844 cases of cryptosporidiosis were reported between 1 November 2005 and 29 May 2006. From November, cases of cryptosporidiosis occurred at higher than expected rates: 119 cases were reported in December, 104 in January, 130 in February, 179 in March, 109 in April and 89 to 29 May 2006. The epidemic curve shows a peak of cases in the weeks commencing 27 November and 12 March (Figure 1).

\section{Demographic characteristics}

Of all 844 cases, 397 (47\%) resided in metropolitan Sydney and 53\% in the remainder of NSW. The majority of the cases reported between 1 November 2005 and 31 December 2005 resided in areas outside of metro- politan Sydney (233; 96\%); this number fell to 267 (44\%) among cases reported from 1 January to 29 May 2006. Overall, higher numbers were reported in the Northern Sydney, Hunter, South Eastern Sydney and Northern Rivers Areas (pre-2005 AHS boundaries).

Of all cases, $49 \%$ were male and $51 \%$ female. By age group, $41 \%$ of cases were aged $0-4$ years, $18 \%$ were aged $5-9$ years, $8 \%$ of cases were aged $10-19$ years, $20 \%$ were aged $20-34$ years and $13 \%$ were aged 35 or over.

\section{Reported exposures}

Of the 844 cases, PHU staff interviewed 464 (55\%) about possible exposures. The most frequent exposures reported by cases in the two weeks before onset of signs and symptoms were: pet contact $(59 \%)$, swimming $(55 \%)$, preschool attendance (26\%), drinking untreated water (20\%) (of whom 67\% drank tank water), other animal contact (21\%) and visiting a farm (18\%). Almost one third (30\%) of cases reported contact with either another possible or notified case. The usual drinking water source at home was reported as town water for the majority of cases (75\%), but $11 \%$ also reported drinking tank water at home; $5 \%$ of cases also reported drinking tank water at work or another place.

Over time, the number of cases reporting specific exposures changed (Figure 2). Swimming remained an important exposure throughout the period. The proportion of cases that reported swimming as an exposure peaked in January (58\%) and decreased sharply in April (10\%). Farm visits and contact with farm animals were more prominent exposures in the earlier stages of the outbreak (in November $2005,21 \%$ reported contact with farm animals or visiting a farm), although some cases also reported these exposures in

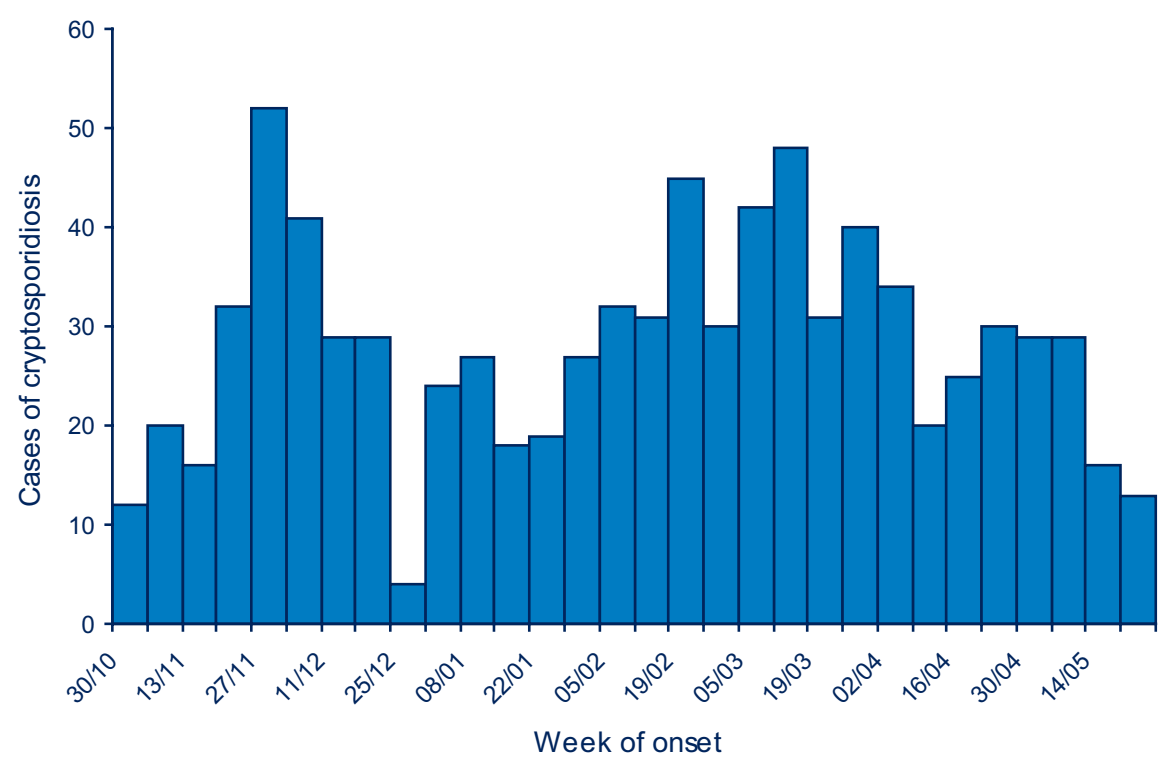

Figure 1. Cases of cryptosporidiosis notified in NSW, 30 October 2005 to 27 May 2006 (by onset date). Source: NSW Health Notifiable Diseases Database 


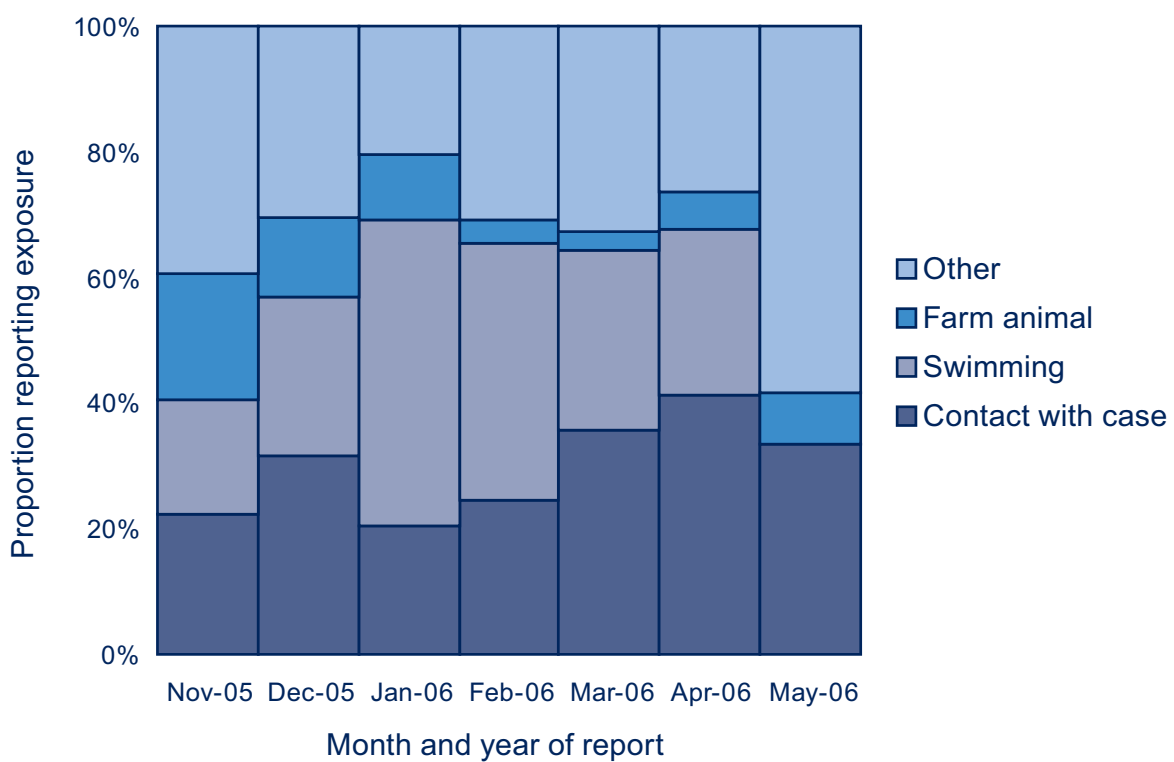

Figure 2. Exposures of cryptosporidiosis cases (as proportion of total) in NSW by month of report, 1 November 2005 to 29 May 2006 (only one exposure reported for each case)

December (8\%) and January (7\%). In November, a small number of cases (six) reported direct contact with scouring calves on their properties. The proportion of cases that reported contact with a possible or notified case peaked in March (42\%), and varied from $20 \%$ in January to $42 \%$ in March. Preschool attendance was also reported consistently throughout the period ( $15 \%$ on average), with a lower proportion of cases reported in December and January as could be expected in holiday periods.

Prior to December 2005, the majority of cases were outside of metropolitan Sydney and in 2006, the proportion fell to under half. Also, prior to December 2005, a greater proportion of cases reported a farm visit or contact with farm animals than in early 2006 and swimming as an exposure was reported by a greater proportion of people in December and January.

\section{Comment}

In this outbreak a large proportion of cases reported swimming, pet contact, preschool attendance, farm visits, animal contact or contact with another case in their incubation period. The number of cases reporting specific exposures changed over time and may reflect changing risk in the course of the outbreak. Patterns of changing geographical distribution and changing exposures in this outbreak support the hypothesis that much of the outbreak began in rural areas of NSW where contact with farms and animals were risk factors, then over time spread via contamination of swimming pools and person-to-person contact.

A limitation in this analysis is a lack of a comparison group, which makes it difficult to draw causal links between exposures and illness. In addition, in recent years, two species of Cryptosporidium: C. hominis and C. parvum have been recognised to infect people. The laboratory data do not allow us to differentiate between the contributions each of them made in this outbreak. It may be that a high proportion of the cases identified earlier in this outbreak were C. parvum (associated with farm animals) than later (when person-to-person and swimming pool spread predominated in the outbreak).

Cryptosporidiosis transmission can be prevented by careful hand washing, avoidance of drinking contaminated drinking and pool water, and avoidance of swimming in pools while infectious. In light of an outbreak in 2007, the Centre for Health Protection conducted a poster campaign designed to educate swimmers about hygiene and pools.

In future outbreaks it would be useful to gather information on cryptosporidiosis species on cases from differing geographical areas and with different exposures in order to ascertain the relative contribution that the species make in outbreaks, and determine the geographical distribution of the two species.

\section{References}

1. Heymann DL. Control of Communicable Diseases Manual 18th ed. Washington DC: American Public Health Association, 2004.

2. Xiao L, Fayer R, Ryan U, Upton SJ. Cryptosporidium taxonomy: Recent advances and implications for public health. Clin Microbiol Rev 2004; 17(1): 72-97. doi:10.1128/CMR.17.1.72-97.2004

3. Caccio SM, Thompson RCA, McLauchlin J, Smith HW. Unravelling Cryptosporidium and Giardia epidemiology. 
Trends Parasitol 2005; 21(9): 430-7. doi:10.1016/j.pt.2005.06.013

4. Puech MC, McAnulty JM, Lesjak M, Shaw N, Heron L, Watson JM. A statewide outbreak of cryptosporidiosis in New South Wales associated with swimming at public pools. Epidemiol Infect 2001; 126(3): 389-96. doi:10.1017/S0950268801005544

5. Black M, McAnulty J. The investigation of an outbreak of cryptosporidiosis in New South Wales in 2005. N S W Public Health Bull 2006; 17(5-6): 76-9.

6. Lemmon JM, McAnulty JM, Bawden-Smith J. Outbreak of cryptosporidiosis linked to indoor swimming pool. Med J Aust 1996; 165(11-12): 613-6.
7. Stafford R, Neville G, Towner C. McCall B. A community outbreak of Cryptosporidium infection associated with a swimming pool complex. Commun Dis Intell 2000; 24(8): 236-9.

8. NSW Department of Health. NetEpi Case Manager Quick Reference Guide. At https://wwwepi3.health.nsw.gov.au/patontraining/help/help.html\#login, accessed 15 February, 2007.

9. SAS Institute. The SAS system for Windows version 8.02. Cary, NC: SAS Institute, 2003.

Erratum. In the Communicable Diseases Report for January and February 2007 (N S W Public Health Bull 2007; 18(3-4):69) the following correction should be made.

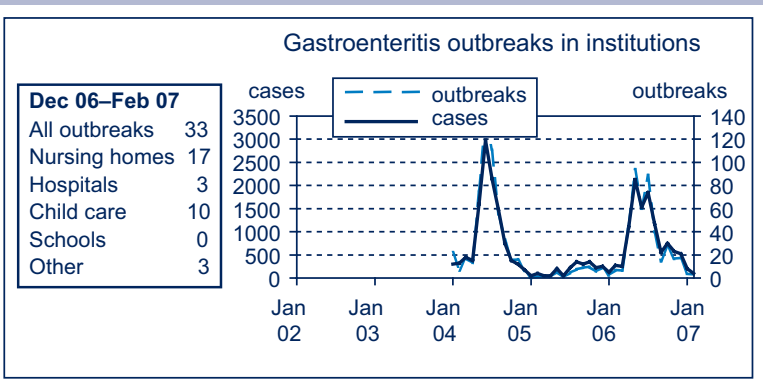


Figure 3. Reports of selected communicable diseases, NSW, Jan 2002 to June 2007, by month of onset.

Preliminary data: case counts in recent months may increase because of reporting delays.

Laboratory-confirmed cases only, except for measles, meningococcal disease and pertussis.

BFV, Barmah Forest virus infections; RRV, Ross River virus infections; Lab Conf, laboratory confirmed;

Men Gp C and Gp B, meningococcal disease due to serogroup C and serogroup B infection; Other/unk, other or

unknown serogroups. NB: multiple series in graphs are stacked, except gastroenteritis outbreaks.

Outbreaks are more likely to be reported by nursing homes and hospitals than by other institutions.
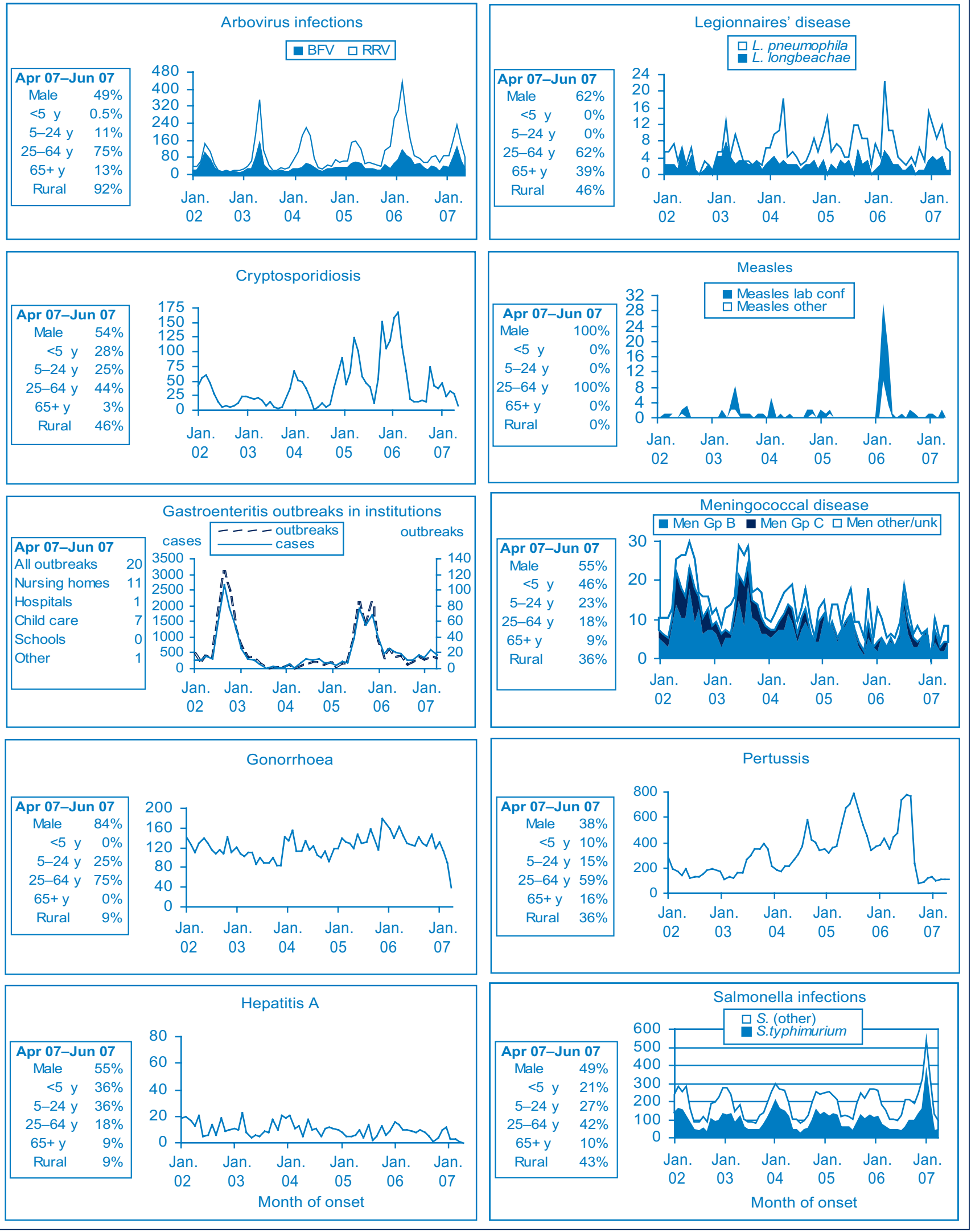
Table 1. Reports of notifiable conditions received in May 2007 by Area Health Services

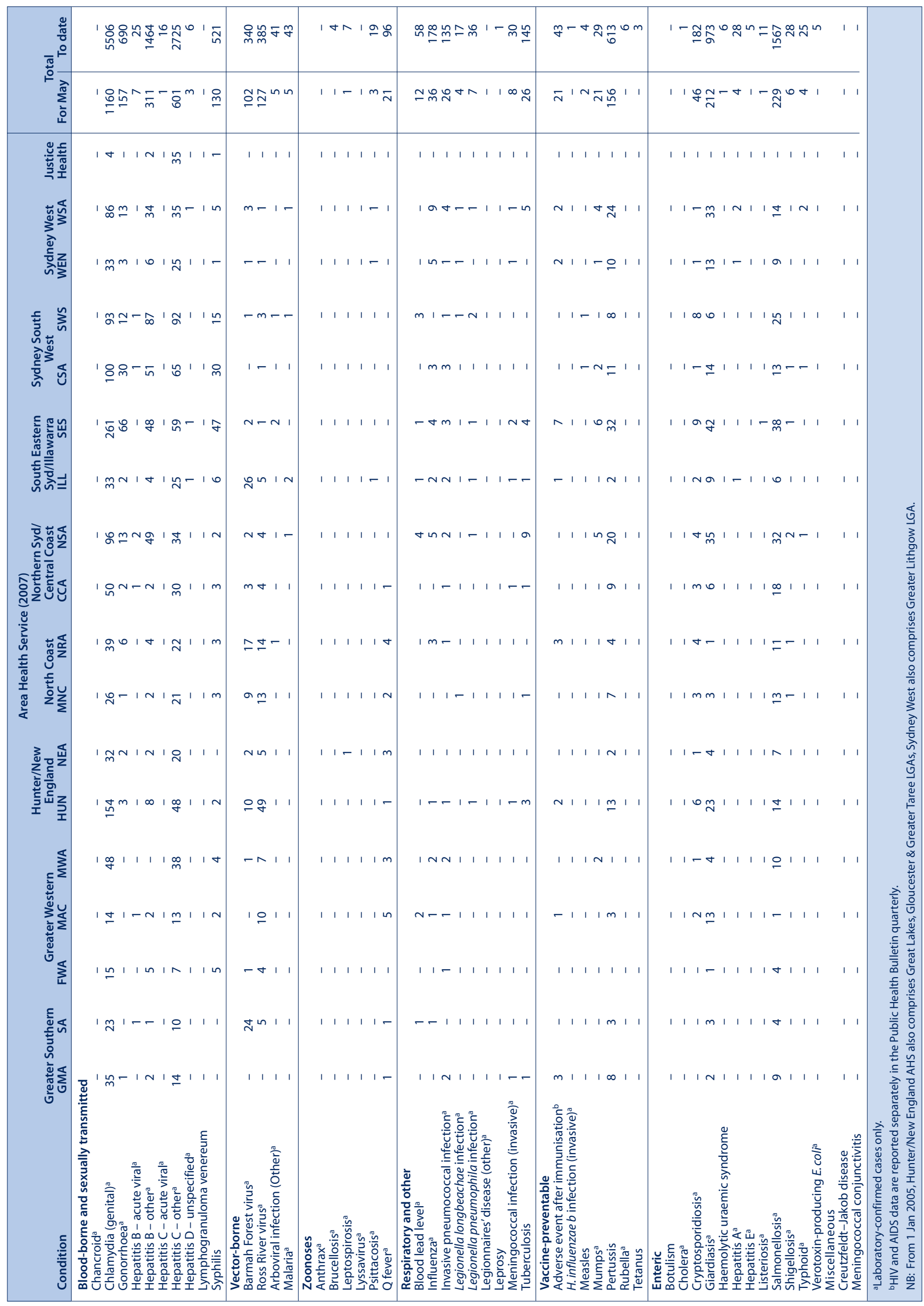


Table 2. Reports of notifiable conditions received in June 2007 by Area Health Services

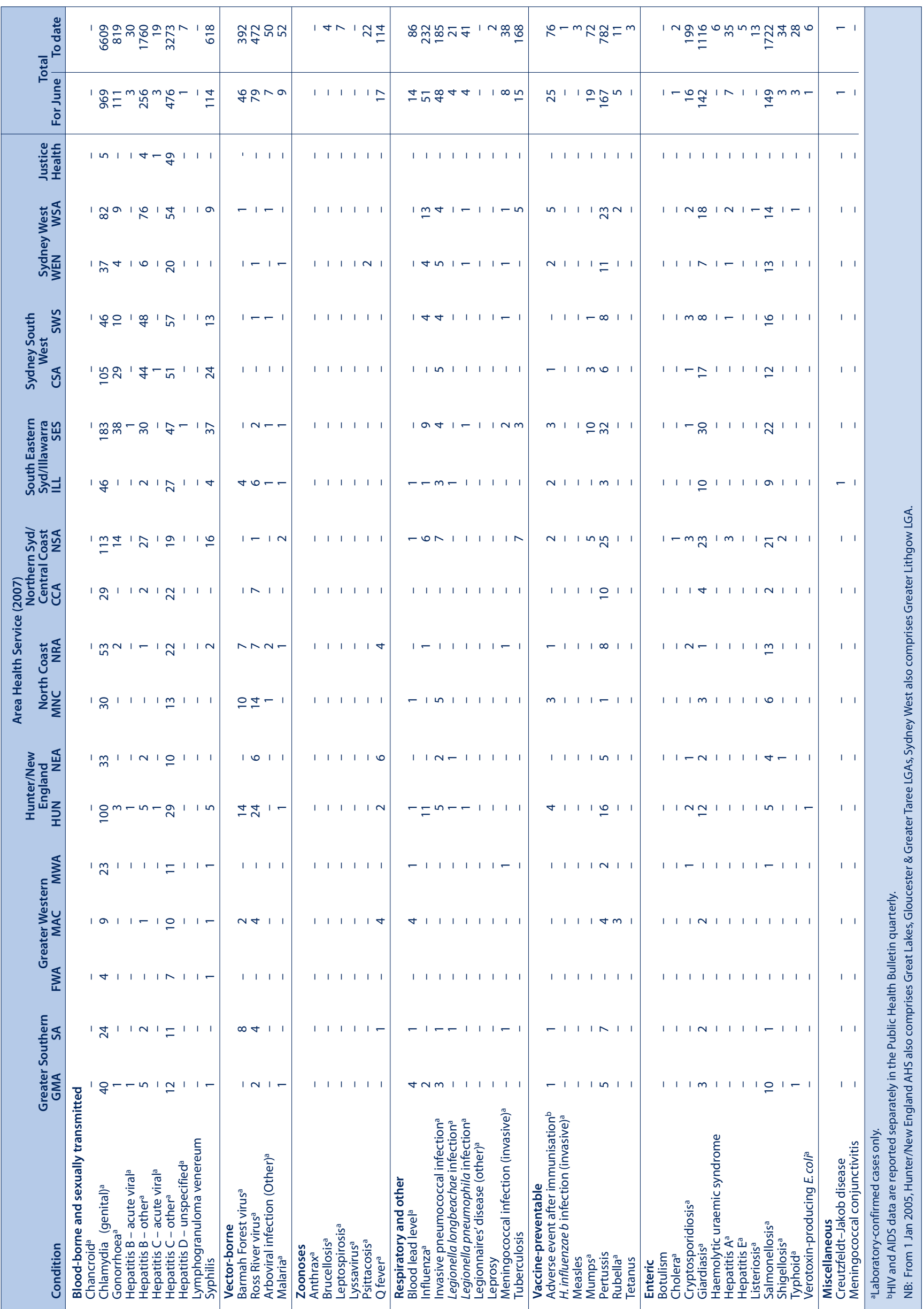

